\title{
Disrupted development and altered hormone signaling in male Padi2/Padi4 double knockout mice
}

Kelly L. Sams ${ }^{1}$, Chinatsu Mukai ${ }^{1}$, Brooke A. Marks ${ }^{1}$, Chitvan Mittal ${ }^{1}$, Elena Alina Demeter $^{2}$, Sophie Nelissen ${ }^{2}$, Jennifer K. Grenier ${ }^{2,3}$, Ann E. Tate ${ }^{2,3}$, Faraz Ahmed ${ }^{2,3}$, Scott A. Coonrod ${ }^{1,2^{*}}$

${ }^{1}$ Baker Institute for Animal Health, College of Veterinary Medicine, Cornell University

${ }^{2}$ Department of Biomedical Sciences, College of Veterinary Medicine, Cornell University

${ }^{3}$ Transcriptional Regulation and Expression Facility, Department of Biomedical Sciences, Cornell University

*Correspondence: sac269@cornell.edu

\section{ABSTRACT}

Peptidylarginine deiminase enzymes (PADs) convert arginine residues to citrulline in a process called citrullination or deimination. Recently, two PADs, PAD2 and PAD4, have been linked to hormone signaling in vitro and the goal of this study was to test for links between PAD2/PAD4 and hormone signaling in vivo. Preliminary analysis of Padi2 and Padi4 single knockout (SKO) mice did not find any overt reproductive defects and we predicted that this was likely due to genetic compensation. To test this hypothesis, we created a Padi2/Padi4 double knockout (DKO) mouse model and tested these mice for a range of reproductive defects. Results from controlled breeding trials found that DKO breeding pairs appeared to take longer to have their first litter than WT controls and that DKO male weanlings weighed significantly less than their WT counterparts. Additionally, DKO males took significantly longer than WT males to reach puberty and had lower serum testosterone levels. Furthermore, DKO males had smaller testes than WT males with increased rates of germ cell apoptosis. The DKO mouse model provides a new tool for investigating PAD function and outcomes from our studies provide the first in vivo evidence linking PADs with hormone signaling.

KEY WORDS: PAD2, PAD4, hormone signaling, estrogen receptor, androgen receptor

\section{INTRODUCTION}

Peptidylarginine deiminases (PADs or PADIs) are a family of calcium-dependent enzymes that post-translationally convert positively charged arginine residues to neutrally charged citrulline in a process called citrullination or deimination. The loss of 
charge on target arginine residues following citrullination can significantly alter the target protein's tertiary structure and affect protein-protein and protein-DNA/RNA interactions (S. Wang \& Wang, 2013). There are five PAD family members (PAD1-4 and 6), with each of the isoforms showing some degree of substrate and tissue specificity (Witalison et al., 2015). Functional roles for PADs in mammalian physiology and pathology are diverse and include cellular differentiation, nerve growth, apoptosis, inflammation, early embryonic development, and gene regulation (Vossenaar et al., 2003).

While PAD activity is most closely associated with autoimmune disease (Valesini et al., 2015), previous studies have found that PAD2 and PAD4 are expressed in reproductive epithelial tissues and that their expression is regulated by steroid hormone signaling (Takahara et al., 1992) (Dong et al., 2007). Steroid hormones are essential mediators of many physiological events including reproduction and development (Mani et al., 2012). These hormones carry out their effects by binding to their cognate nuclear receptors which, in turn, bind to hormone response DNA elements and regulate transcription via interaction with a wide range of co-regulators and the basal transcriptional machinery. Given the potential links between PADs and steroid hormones and that histones were found to be targeted by PAD4 for citrullination (Hagiwara et al., 2002), we previously tested whether PAD2 and PAD4 may play a role in estrogen signaling in breast cancer cells by facilitating ER target gene expression via histone citrullination at ER binding sites. Results from our studies found that PAD4 is recruited to the TFF1 promoter (a canonical $\mathrm{ER} \alpha$ binding site) in MCF7 cells following E2 treatment leading to citrullination of histone $\mathrm{H} 4$ arginine 3 (Y. Wang et al., 2004). Additionally, we found that PAD2 interacts with ER in an estrogen-dependent manner and that estrogen (E2) treatment activates PAD2 leading to citrullination of histone $\mathrm{H} 3$ arginine 26 (H3Cit26) at ER binding sites (L. Wang et al., 2017; Zhang et al., 2012). We then demonstrated by ChIPSeq that $\sim 95 \%$ of ER binding sites throughout the genome are citrullinated at H3Cit26 within 5-10 minutes of E2 treatment (Guertin et al., 2014). We also found that PAD inhibitors potently blocked ER binding at Estrogen Response Elements (EREs) and prevented ER target gene activation (Zhang et al., 2012). Interestingly, another group recently found that PAD2 plays a role in androgen receptor $(A R)$ signaling by facilitating nuclear import of $A R$ and promoting $A R$ target gene activation via histone citrullination at AR binding sites (L. Wang et al., 2017). This finding suggests that the role of PADs in regulating hormone-mediated transcription may be broader than we had previously realized. Importantly, to date, there have not been any published reports linking PADs to hormone signaling in animal models and this lack of in vivo data has likely limited research into this area.

To address this gap in knowledge, we began testing for links between PADs and hormone signaling in vivo by determining whether Padi2 and Padi4 single knockout (SKO) mouse lines displayed overt reproductive defects. Initial studies with these mice 
found that both PAD2 and PAD4 mice are largely normal with no clear reproductive defects (data not shown). Given the greater than 59\% amino acid homology between PAD family members and that $P$ adi genes are closely clustered together at chromosome 1p35-36 in humans and chromosome 4E1 in mice (Vossenaar et al., 2003) (Briot et al., 2020), we predicted that the lack of a phenotype in the SKO lines was due to genetic compensation by other Padis. In order to test this hypothesis, we generated a Padi2/Padi4 double knockout mouse (DKO) line by deleting Padi4 from our existing Padi2 knockout (P2KO) mouse line using CRISPR/CAS9 technology. We demonstrate that, as opposed to the SKO lines, the Padi2/Padi4 DKO mice display a range of reproductive defects, and the goal of this report is to document the DKO phenotype in males. Outcomes from our studies show that DKO males displayed a reduced pup and weanling weight, delayed puberty, reduced testis size, increased rates of apoptosis during spermatogenesis, and altered circulating hormone levels. Our results provide the first in vivo evidence that PADs play a role in male reproduction, potentially via their role in hormone signaling.

\section{RESULTS and DISCUSSION}

\section{Generation of Padi2/Padi4 double knockout mice}

Our strategy for generating the Padi2/Padi4 DKO mouse line is shown in Figure 1a. Padi2-null males and females were bred to generate Padi2-null zygotes and these embryos were then injected with a Padi4 sgRNA construct and transferred to wild type recipient females to generate founder pups. The Padi4 sgRNA construct was designed to target Exon 1 of the Padi4 gene (Figure 1b). In total, 39 founders were born, with 13 CRISPR-edited founders being identified by heteroduplex PCR, subcloning, and Sanger sequencing. All of the edited founders were heterozygous mutants, with seven of these founders having a frame-shift mutation. Following breeding and selection by genotyping, one homozygous DKO strain was selected for further characterization. This mutant mouse line contained a four nucleotide deletion in exon 1 of Padi4 (Figure 1c) and a predicted premature stop codon (Figure 1d).

Validation of the DKO mutant line is shown in Figure 2. We first confirmed that P2KO and DKO mice lacked Padi2 transcripts. RT-PCR analysis of salivary gland RNA showed that the P2KO and DKO mice lacked a Padi2 PCR product, while the appropriately sized product was seen in the WT sample (Figure 2a). We next tested whether the Padi4 sgRNA targeting site in DKO mice contained a deleted sequence by performing PCR analysis of genomic DNA and then separating the DNA fragments on high percentage polyacrylamide gels. Results showed that the DKO amplicon displayed a reduced molecular weight when compared to the P2KO and WT amplicons (Figure $2 b$ ), suggesting that DKO mice contained the targeted deletion. As expected, no 
amplicon was observed in P4KO mice. The above results suggested that DKO mice lacked both Padi2 and Padi4 transcripts. We then performed Western blot analysis of WT, P2KO, P4KO and DKO spleen lysates to test if the DKO mice also lacked PAD2 and PAD4 protein. We probed the protein lysates with anti-PADI2 antibodies and found that, while the antibody was reactive with appropriately sized $\sim 75 \mathrm{kDa}$ bands in the WT and P4KO lysates, the antibody did not react with similarly sized bands in the P2KO and DKO mice (Figure 2c, Supplemental 1). Likewise, the anti-PADI4 antibody was reactive with $\sim 75 \mathrm{kDa}$ bands in the WT and P2KO lysates but was not reactive with similarly sized bands in the P4KO and DKO lysates (Figure 2c). These results suggest that the Padi2/Padi4 DKO strain does not express mature PAD2 and PAD4 proteins.

\section{Effect of Padi2/Padi4 DKO on fertility parameters}

In order to begin testing for links between PADs and reproductive function, we first performed a controlled breeding trial to test whether a range of reproductive parameters were affected by DKO. Seven-week old DKO males were paired with similarly aged DKO females and the pairs were monitored daily for 63 days. Birth dates, litter size, sex ratio, pup weights, and weanling weights were evaluated and recorded. Results showed that, over the initial two months, DKO pairs consistently appeared to lag behind WT pairs in their time to first litter (Figure $3 a$, data not statistically significant). We also found that the sex ratio of DKO offspring was skewed ( 0.85 male:female) but did not deviate significantly from the expected equal ratio ( $\chi 2$ test, $n=243, p=0.22$ ), and that the average DKO litter size was similar to that of WT mice $(n=4, p=0.5)$. Interestingly, however, we also observed that 2-day old DKO pups weighed significantly less than WT pups (Figure $3 b, n=4, p=0.004$ ) and that DKO male weanlings weighed significantly less than WT male weanlings (Figure $3 c-d, n=21,45, p=0.003$ ). Taken together, results from these studies suggest that loss of PAD2 and PAD4 suppresses fertility and affects offspring weight. Another larger fertility trial is currently being planned to test whether increasing the sample size will yield statistically significant differences in the fertility rate. Regarding the effect of DKO on pup weight, previous studies have shown that pup weight is also significantly reduced in androgen receptor knockout (ARKO) mice (Yeh et al., 2002). Given the growing links between PADs and hormone signaling, we hypothesize that the effect of DKO on both fertility and pup weight is because hormone signaling is altered in these mice.

\section{Effect of Padi2/Padi4 DKO on pubertal onset}

Pubertal onset is thought to be primarily driven by increased testosterone production in males and one well established external marker of pubertal development in rodents is preputial separation (PS) (Gaytan et al., 1988; Hoffmann, 2018; Korenbrot et al., 1977). Therefore, as a further test of the hypothesis that hormone signaling is disrupted in DKO 
males, we next documented the timing of PS in WT and DKO males. Results show that DKO males $(n=46, m=31)$ took an average of three days longer than WT males $(n=49$, $m=28$ ) to undergo PS ( $p<0.00001$, Figure 4). Given that PS is known to be an androgen-dependent process (Korenbrot et al., 1977; Yoshimura et al., 2005) and to be strongly linked to hormone signaling along the hypothalamic-pituitary-gonadal (HPG) axis (Handelsman, 2020; Keene et al., 2002), these results further support the hypothesis that hormone signaling is altered in DKO males. Additionally, these observations suggest that PAD2 and PAD4 play an important role in androgen-driven sexual development in males.

\section{Effect of Padi2/Padi4 DKO on serum hormone levels}

As a more direct test of the hypothesis that hormone signaling is defective in DKO mice, we next measured serum testosterone (T), luteinizing hormone (LH), and follicle stimulating hormone (FSH) in WT and DKO males. Results showed that levels of T, LH, and FSH differed significantly between DKO and WT at various times $(n=5$ per time point). Although highly variable, serum testosterone levels were lower in DKO males than WT males at all time-points, most significantly at day 48 ( $p=0.05$, Figure 5). In contrast, FSH and LH levels were statistically similar between DKO and WT at the earlier time points, but by day 50 both hormones were significantly higher in DKO sera $(p<0.03)$. Serum testosterone levels are difficult to accurately measure in mice, likely due to the fact that mice do not express significant levels of sex-hormone binding globulin (Jänne et al., 1998), therefore, we were encouraged by the observation that serum $\mathrm{T}$ levels in DKO mice were lower than WT mice at each time point evaluated, reaching significance at day 48. Given that $\mathrm{T}$ is known to negatively regulate $\mathrm{FSH}$ and LH secretion (Hayes et al., 2001), we were also encouraged by the observation that, on the day when T levels appeared to be lowest in DKO males (day 50), FSH and LH levels were correspondingly elevated. Importantly, studies with ARKO mice also found that serum T levels are lower in mutant mice compared to WT mice (Yeh et al., 2002). Given this observation, our findings lend support to the prediction that PAD enzymes play an important role in androgen signaling in vivo.

\section{Effect of Padi2/Padi4 DKO on testis size and histology}

Testis Size. Hormone signaling within the HPG axis not only regulates pubertal onset, but also regulates body growth (Corre et al., 2016) and testis size (Huh et al., 2021; Miyaso et al., 2021). In support of this observation, previous studies with ARKO mice found that both pup size and testis weight are reduced in mutant mice (Yeh et al., 2002). As a further test for potential associations between PADs and hormone signaling, we next investigated whether the total body weight and testis weight was altered in 90-day old adult mice. Results showed that, while there was no difference in 
total body weight between 90-day old WT $(m=27.4 \mathrm{~g})$ and DKO $(m=27.4 \mathrm{~g})$ mice $(\mathrm{n}=5$, $p=1$ ), testis weight was significantly lower in DKO males when compared to WT males $(n=5, p=0.002$, Figure $6 a)$. The finding that DKO testes are significantly smaller than WT testes in adults fits well with the hypothesis that PADs play a role in androgen signaling in males. Given our hypothesis, it is currently unclear why the body weight of adult DKO mice is similar to that of adult WT mice. It is possible that the effect of PAD2/4 DKO on adult male body weight is transient in nature and that other PADs may be able to compensate for PAD2/4 in adults.

Testis Histology. The observation that DKO testes are smaller than WT testes raised the possibility that testicular histology may also be defective in DKO males. To test this hypothesis, FFPE sections of adult testes were stained with H\&E and scored for cellular morphology by a board-certified pathologist (Figure 6b-e). Results of this analysis found that there were variable degrees of germ cell apoptosis in the DKO testes when compared to WT testes, with this apoptosis mostly involving spermatogonia and spermatocytes (Supplemental 2). More specifically, apoptosis (mild/moderate grade) in spermatocytes was noted in 5 out of 5 DKO mice compared to only 2 out of 5 WT samples (Figure 6c). Apoptosis (mild grade) of spermatogonia was noted in 4 out of 5 DKO mice testes compared to only 1 out of 4 WT mice (Figure 6d). Five of 5 DKO testes also displayed moderate grade residual bodies when compared with WT testes, which only displayed mild grade residual bodies (Figure 6e). Sertoli, Leydig cells and interstitium were overall unremarkable. Interestingly, the increased rate of apoptosis and residual bodies did not appear to result in a decrease in sperm production with the lumen of most tubules being lined with histomorphologically unremarkable elongated spermatids and spermatozoa (data not shown). Interestingly, in ARKO mice, spermatogenesis was arrested at the pachytene stage of meiosis and there was an increase in apoptotic-like bodies within the tubules (Yeh et al., 2002) suggesting that androgen signaling is critical for normal spermatogenesis. Taken together, these results support the hypothesis that PAD2/4 signaling may play a role in androgen signalingmediated spermatogenesis. The relatively modest effect of DKO on testicular histology and the other observed hormone-mediated phenotypic traits could potentially be due to compensatory effects of other PADs in the DKO males and subsequent studies are planned to test this hypothesis. As an alternate hypothesis, it may be that PAD2/4 is only involved in regulating a portion of androgen-mediated signaling events.

\section{Effect of Padi2/Padi4 DKO on gene expression in the testis}

In order to begin investigating whether PADs are associated with specific signaling pathways in the testis, and to more directly test the hypothesis that PAD2/4 play a role in regulating AR target gene expression, we next carried out RNA-Seq analysis of adult WT, P2KO, and DKO testes. Results show that 22 genes were upregulated and 19 
genes were downregulated in P2KO samples when compared to WT samples $(\mathrm{P}<0.05)$. Additionally, we found that 263 genes were upregulated and 140 genes were downregulated in the DKO samples compared to WT samples $(P<0.05)$. Seventeen of the 22 genes that were significantly upregulated in the P2KO samples were also upregulated in the DKO samples. A comparison of upregulated genes is shown in Figure 7a. The complete lists of DE genes are shown in Supplementals 3 and 4. The 246 genes that were found to be uniquely upregulated in DKO testes were then subjected to DAVID gene ontology analysis. Two hundred and thirty eight genes were identified in the DAVID mouse database and were categorized into three domains: Cellular Components, Molecular Functions, and Biological Processes (Supplemental 5). Interestingly, analysis of the Biological Processes domain finds that a majority of the DE genes are involved in reproductive functions (Figure $7 \mathrm{~b}$ ), supporting the phenotypic traits we observed. In addition, analysis of the Molecular Function domain finds that nearly all of the DE genes appear to be involved in either nucleic acid binding or protein phosphorylation (Figure 7c), thus further supporting the hypothesis that PADs play a role in regulating gene transcription. We next generated a hierarchical dendrogram and heatmap showing the top 100 significantly DE genes using summary counts over all pairwise tests of WT, P2KO, and DKO samples (FDR cut-off 0.05). Results show that there is a good correlation in gene expression levels between replicates for each experimental group. Additionally, the results show that the gene expression profile for the DKO samples is distinctly different from that of the WT samples, while there are some overlapping expression patterns when comparing the DKO and P2KO samples (Figure 8, Supplemental 6).

Interestingly, analysis of the list of genes that are most highly overexpressed in the DKO samples compared to WT and P2KO samples, finds that 3 of the top 10 genes (KIk1b27, Klk1b21, and Klk1b24) are members of the kallikrein gene family. Perhaps the best-known member of this family is prostate-specific antigen (KLK3), whose expression is mainly induced by androgen and is transcriptionally regulated by the androgen receptor (Kim \& Coetzee, 2004). Additionally, the kallikrein gene locus is highly responsive to steroid hormones, having at least 14 functional HREs in this region. In fact, many researchers use kallikreins as markers of hormone receptor activity (Lawrence et al., 2010). Importantly, Klk1b27, Klk1b21, and Klk1b24 are all expressed in leydig cells in rodents and their expression is regulated by androgen (Lawrence et al., 2010). In addition to kallikreins, a number of other genes that were overexpressed in the DKO gene set also appear to be regulated by androgens (data not shown). These findings further support our hypothesis that PADs play an important role in regulating gene expression in the testis, and more specifically, in regulating AR-mediated gene expression.

\section{CONCLUSIONS}


Our previous work with PAD2 and PAD4 in breast cancer cell lines has found that both of these PAD isozymes appeared to regulate ER target gene expression via citrullination of histone residues at ER binding sites. More recent studies have shown that PAD2 also appears to facilitate androgen receptor target gene expression in prostate cancer cells via similar mechanisms. Together, these observations suggest that PAD2 and PAD4 may represent key mediators of hormone signaling in mammals. While these in vitro findings are intriguing, an important next step in linking PADs with hormone signaling is demonstrating that the phenomenon also occurs at the organismal level. While our Padi2 and Padi4 SKO mouse models have failed to show a reproductive phenotype, outcomes from our Padi2/Padi4 DKO studies provide a series of clear associations between PADs and hormone signaling and, to our knowledge, this study is the first to make such genetic links using animal models. Subsequent studies are now being designed to further investigate the direct mechanisms by which PADs are regulating hormone signaling in males. An important part of these future studies will be to localize the expression of both PAD2 and PAD4 in the testis. Recent studies have documented the presence of PAD2 in the testis. For example, Hosokawa et al. showed that PAD2 appears to play an important role in testis development and is specifically expressed in fetal sertoli cells with its expression being regulated by SOX9, a critical mediator of sex determination (Tsuji-Hosokawa et al., 2018). Another recent study found by PCR, western blotting, and IHC that PAD2 is expressed in sperm during the first wave of spermatogenesis and appears to localize to the acrosomal region of the sperm head (Sahadevan \& Kumar, 2021). There are currently no reports of PAD4 localization to the testes, however, IHC analysis of PAD4 staining in the testis from the Human Protein Atlas website (Pontén et al., 2008) does show anti-PAD4 staining (moderate intensity) specifically in Leydig cells. Based on these reports, we predict that the phenotypic effects of DKO that we have observed in this study are due to the lack of PAD2 and PAD4 activity in the testis. Subsequent studies will be carried out to more precisely localize each PAD to specific testicular cell types and to more directly test the hypothesis that PADs facilitate androgen receptor-mediated target gene expression in the testis.

\section{MATERIALS AND METHODS}

\section{Animals}

All mice used in this work were on an FVB/NJ background and were maintained as inbred homozygous lines. The mice were housed in an AAALAC certified facility, with 12 hours on/off light cycle, temperature control and food and water ad libitum. All procedures were approved by Cornell University's Animal Care and Use Committee (IACUC) and in compliance with the US National Research Council's Guide for the Care and Use of Laboratory Animals, the US Public Health Service's Policy on Humane Care 
and Use of Laboratory Animals, and Guide for the Care and Use of Laboratory Animals. Breedings were recorded and lineages tracked with Softmouse Colony Management Software (https://www.softmouse.net). Euthanasia was performed by $\mathrm{CO}_{2}$ inhalation in accordance with AVMA approved practice.

\section{Generation of transgenic single knockout mice}

Padi2-null mice (FVB/NJ background) were generated using gene trap technology at the Texas A\&M Institute for Genomic Medicine (Hansen et al., 2008). For the gene trap, 163 nucleotides of exon 1 in Padi2 (from the ATG initial codon to intron 1-2) were replaced by the LacZ and Neomycin coding sequence (Supplemental figure). These mice were genotyped in a multiplex mixture using Padi2 WT primers: Forward 5' CTTATTCCTGGGAGCAGCAC 3', Reverse 5' CTCTCTCTCGGCTCACCTGT 3' and KO primers: LacZ-Neo Forward 5' GATCGGCCATTGAACAAGAT 3', Reverse 5' GTAGCCGGATCAAGCGTATG 3'.

Padi4-null mice (FVB/NJ background) were generated using Regeneron's VelociGene technology. The genomic sequence of Padi4 from the ATG initial codon to approximately $100 \mathrm{bp}$ downstream of the TGA stop codon was replaced in frame (with respect to the Padi4 initiation codon) with a Lox-flanked LacZ and neomycin coding sequence (Supplemental figure). These mice are genotyped using Padi4 primers: Forward 5' CTCGCCTGGCACTATAAAGG 3' and Reverse 5' AGGATCTCTGCCCCTCTCAC 3'.

\section{Generation of Padi2/Padi4 double knockout mice}

Padi2-null mice were used for IVF and CRISPR/Cas9 RNA injection was performed at Cornell's Stem Cell \& Transgenic Core Facility. Cas9 mRNA and Padi4 sgRNA targeting exon 1 of the Padi4 gene was injected into the pronucleus of Padi2 KO embryos and the embryos were cultured to the two-cell stage and transferred into pseudopregnant recipient female mice (Singh et al., 2015; Singh \& Schimenti, 2015). Thirty nine founders were produced and 13 edited founders were then identified by heteroduplex PCR (Zhu et al., 2014). Briefly, genomic DNA was extracted from punched ear tissue and evaluated by PCR using the Padi4 primer set noted above. Standard PCR conditions for GoTaq (Promega) were used as follows: $95^{\circ} \mathrm{C}$ for $5 \mathrm{~min} ; 94^{\circ} \mathrm{C}$ for 30 s, $59^{\circ} \mathrm{C}$ for $30 \mathrm{~s}, 72^{\circ} \mathrm{C}$ for $30 \mathrm{~s}$ for 35 cycles; $72^{\circ} \mathrm{C}$ for $5 \mathrm{~min}$ followed by 5 minutes of denaturation at $95^{\circ} \mathrm{C}$. PCR products were loaded onto a $12 \%$ polyacrylamide TBE gel. Positive heteroduplex bands were purified and subjected to TA cloning for Sanger sequencing. All 13 edited founders were found to be heterozygous mutants, with 7 founders being identified as having a frameshift mutation either by insertion or deletion (Figure 1b). One heterozygous founder with a four nucleotide deletion was bred with 
Padi2 knockout mice, and their heterozygous offspring were bred to create a homozygous DKO line.

\section{Authentication of Padi2/Padi4 double knockout mice}

RT-PCR. Total RNA was extracted from mouse salivary glands using Tri-Reagent (Molecular Research Center \#TR118) and cDNA was created with a High Capacity RNA-to-cDNA Kit (applied biosystems \#4387406) according to manufacturers' instructions. PCR amplification of Padi2 was accomplished under standard cycling conditions with GoTaq (Promega \#M3001) using the following Padi2 primers: Forward 5' TCCCCTCGAGATGGAAACCT 3' and Reverse 5' CTCCCAGGCCCTTGAACATAA 3'. PCR products were run on a $2 \%$ Agarose gel for visualization.

Western blot. Tissue lysate was prepared from splenic tissue and resolved by SDSPAGE followed by transfer to a PVDF membrane. The membranes were incubated overnight with primary antibodies diluted in $1 \%$ BSA-TBST at $4^{\circ} \mathrm{C}$ using the following concentrations: anti-PAD4 (1:1000; Abcam; ab214810), anti-PAD2 (1:1000, protein tech, 12110-1-AP) and anti-actin (1:1000, Abcam, ab-8227). The primary antibodies were detected with HRP-conjugated secondary antibodies and were exposed to ECL reagents.

\section{Breeding Trials}

Seven-week old virgin mice were paired in the following groups: DKOxDKO, DKOxWT, WTxDKO or WTxWT ( $n=5-6)$. Pairs were monitored daily for 63 days and birth dates, litter sizes, sex ratios, 2-day old pup weights, and weanling weights were recorded. Statistical analyses were carried out using Microsoft Excel and Social Science Statistics (socscistatistics.com) calculators for unpaired Student's t-test, ANOVA, ?2 and Goodness of Fit tests with significance set at $p<0.05$.

\section{Growth and development}

Male weanlings were housed singly (to avoid intra-cage dominance issues), weighed and monitored daily for preputial separation as previously described (Hoffmann, 2018). Mice were euthanized for tissue harvest at ages 46, 48, 50 and 90 days old. At each of these time points, five mice were euthanized by $\mathrm{CO}_{2}$ inhalation and blood was collected by cardiac puncture, allowed to clot at room temperature for 90 minutes, spun at $2000 \mathrm{~g}$ for 15 minutes, and the serum was snap frozen and saved for hormone analysis.

Serum testosterone, FSH, and LH levels were quantified in duplicate by ELISA at the University of Virginia Ligand Assay Core. Testes were collected and weighed individually. Results were analyzed with unpaired t-tests with significance set at $p<0.05$. 


\section{Histological evaluation}

Testis, epididymis, vas deferens, seminal vesicle and splenic tissues were collected at 90 days from 5 animals from each of the following strains: wild type (FVB), P2KO, $\mathrm{P} 4 \mathrm{KO}$, and DKO. Collected tissues were fixed in $10 \%$ formalin solution, embedded in paraffin, sectioned at $5 \mu \mathrm{m}$, and stained with hematoxylin and eosin (H\&E). Initial evaluation revealed epididymis, vas deferens, seminal vesicles, and spleen within normal limits based on previously published observations (Creasy et al., 2012; Meistrich \& Hess, 2013), therefore, only testis sections were evaluated further. The noted changes were scored on a scale of 0 to 3 according to the following criteria: $0=$ absent to affecting less than $5 \%$ of the tissue; 1 = mild change, affecting $5-25 \%$ of the tissue; 2 $=$ moderate change, affecting $25-50 \%$ of the tissue; $3=$ severe change, affecting $>50 \%$ of the tissue (Supplemental 2).

\section{RNA sequencing}

Mouse testes were dissected from two age-matched adult mice per strain (WT, P2KO, and DKO) and snap-frozen immediately following euthanasia. Total RNA extraction was performed using TRI reagent (Molecular Research Center, Inc. OH) according to the manufacturer's protocol. The RNA concentration and purity were measured by Nanodrop and Qubit (Thermo Scientific, USA).

RNA-seq experiments were performed in the Transcriptional Regulation \& Expression Facility at Cornell University. RNA integrity was confirmed on a Fragment Analyzer (Agilent) and RNAseq libraries were prepared using the NEB Ultra II Directional RNA library Prep kit (New England Biolabs), with 1 ug total RNA input and initial poly(A) selection. Illumina sequencing was performed on a NovaSeq to generate at least $20 \mathrm{M}$ $2 \times 150$ paired end reads per sample. The raw fastq reads were first processed with the trim-galore (Barbraham Institute, https://www.bioinformatics.babraham.ac.uk/projects/trim galore/ ) package to trim for low quality reads, noisy short fragments, and adapter sequence. The filtered reads were then aligned to the GRCm38 reference genome $(\mathrm{mm} 10)$ with ENSEMBL annotations via STAR (Dobin et al., 2013) using parameters [--outSAMstrandField intronMotif , -outFilterlntronMotifs RemoveNoncanonical, --outSAMtype BAM SortedByCoordinate, -quantMode GeneCounts]. Differential gene expression analysis was performed by DESeq2 (Love et al., 2014; Varet et al., 2016). A heatmap and hierarchical clustering dendrogram of differentially expressed genes was produced using Morpheus (https://software.broadinstitute.org/morpheus ). Venn diagrams were produced with BioVenn (Hulsen et al., 2008).

Gene ontology classification analysis was performed using DAVID v6.8, a web-based bioinformatics resource for functional genomics analysis to identify biological processes, 
molecular functions, and cellular components (Huang et al., 2009a, 2009b). 246 uniquely upregulated genes were identified when comparing DKO to WT using an FDR $<0.05 .238$ of these genes were identified in the DAVID database and used for subesquent analysis.

\section{ACKNOWLEDGMENTS}

The authors acknowledge the excellent skills and technology provided by Cornell's Stem Cell \& Transgenic Core Facility to create our double knockout mice. We also thank the Baker Institute husbandry technicians for the outstanding care of the animals used in this research.

\section{AUTHOR CONTRIBUTIONS}

Experimentation was conceived and conducted by KLS, CM, BAM and SAC. Breeding and phenotype analysis by KLS and SAC. Histological scoring by EAD and SN. RNAseq and analysis by JKG, AET, and FA, with visualization by CM and KLS. All authors contributed to writing and editing the manuscript and have no conflicts of interest to declare.

\section{REFERENCES}

Briot, J., Simon, M., \& Méchin, M.-C. (2020). Deimination, Intermediate Filaments and Associated Proteins. International Journal of Molecular Sciences, 21(22), 8746. https://doi.org/10.3390/ijms21228746

Corre, C., Shinoda, G., Zhu, H., Cousminer, D. L., Crossman, C., Bellissimo, C., Goldenberg, A., Daley, G. Q., \& Palmert, M. R. (2016). Sex-specific regulation of weight and puberty by the Lin28/let-7 axis. The Journal of Endocrinology, 228(3), 179-191. https://doi.org/10.1530/JOE-15-0360

Creasy, D., Bube, A., Rijk, E. de, Kandori, H., Kuwahara, M., Masson, R., Nolte, T., Reams, R., Regan, K., Rehm, S., Rogerson, P., \& Whitney, K. (2012). Proliferative and Nonproliferative Lesions of the Rat and Mouse Male Reproductive System. Toxicologic Pathology, 40(6_suppl), 40S-121S. https://doi.org/10.1177/0192623312454337

Dobin, A., Davis, C. A., Schlesinger, F., Drenkow, J., Zaleski, C., Jha, S., Batut, P., Chaisson, M., \& Gingeras, T. R. (2013). STAR: Ultrafast universal RNA-seq aligner. Bioinformatics, 29(1), 15-21. https://doi.org/10.1093/bioinformatics/bts635

Dong, S., Zhang, Z., \& Takahara, H. (2007). Estrogen-Enhanced Peptidylarginine Deiminase Type IV Gene (PADI4) Expression in MCF-7 Cells Is Mediated by Estrogen Receptor-a-Promoted Transfactors Activator Protein-1, Nuclear FactorY, and Sp1. Molecular Endocrinology, 21(7), 1617-1629. 
https://doi.org/10.1210/me.2006-0550

Gaytan, F., Bellido, C., Aguilar, R., \& Aguilar, E. (1988). Balano-Preputial Separation as an External Sign of Puberty in the Rat: Correlation with Histologic Testicular Data/Balano-praeputial-Trennung als ein äußeres Zeichen der Pubertät für die Ratte: Korrelation mit histologischen Daten. Andrologia, 20(5), 450-453. https://doi.org/10.1111/j.1439-0272.1988.tb00724.x

Guertin, M. J., Zhang, X., Anguish, L., Kim, S., Varticovski, L., Lis, J. T., Hager, G. L., \& Coonrod, S. A. (2014). Targeted H3R26 Deimination Specifically Facilitates Estrogen Receptor Binding by Modifying Nucleosome Structure. PLOS Genetics, 10(9), e1004613. https://doi.org/10.1371/journal.pgen.1004613

Hagiwara, T., Nakashima, K., Hirano, H., Senshu, T., \& Yamada, M. (2002).

Deimination of Arginine Residues in Nucleophosmin/B23 and Histones in HL-60 Granulocytes. Biochemical and Biophysical Research Communications, 290(3), 979-983. https://doi.org/10.1006/bbrc.2001.6303

Handelsman, D. J. (2020). Testosterone, Spermatogenesis, and Unravelling the Mysteries of Puberty. Endocrinology, 161(9), bqaa120.

https://doi.org/10.1210/endocr/bqaa120

Hansen, G. M., Markesich, D. C., Burnett, M. B., Zhu, Q., Dionne, K. M., Richter, L. J., Finnell, R. H., Sands, A. T., Zambrowicz, B. P., \& Abuin, A. (2008). Large-scale gene trapping in C57BL/6N mouse embryonic stem cells. Genome Research, 18(10), 1670-1679. https://doi.org/10.1101/gr.078352.108

Hayes, F. J., DeCruz, S., Seminara, S. B., Boepple, P. A., \& Crowley, W. F., Jr. (2001). Differential Regulation of Gonadotropin Secretion by Testosterone in the Human Male: Absence of a Negative Feedback Effect of Testosterone on FollicleStimulating Hormone Secretion1. The Journal of Clinical Endocrinology \& Metabolism, 86(1), 53-58. https://doi.org/10.1210/jcem.86.1.7101

Hoffmann, H. M. (2018). Determination of Reproductive Competence by Confirming Pubertal Onset and Performing a Fertility Assay in Mice and Rats. JoVE (Journal of Visualized Experiments), 140, e58352. https://doi.org/10.3791/58352

Huang, D. W., Sherman, B. T., \& Lempicki, R. A. (2009a). Systematic and integrative analysis of large gene lists using DAVID bioinformatics resources. Nature Protocols, 4(1), 44-57. https://doi.org/10.1038/nprot.2008.211

Huang, D. W., Sherman, B. T., \& Lempicki, R. A. (2009b). Bioinformatics enrichment tools: Paths toward the comprehensive functional analysis of large gene lists. Nucleic Acids Research, 37(1), 1-13. https://doi.org/10.1093/nar/gkn923

Huh, K., Nah, W. H., Xu, Y., Park, M. J., \& Gye, M. C. (2021). Effects of Recombinant Human Growth Hormone on the Onset of Puberty, Leydig Cell Differentiation, Spermatogenesis and Hypothalamic KISS1 Expression in Immature Male Rats. The World Journal of Men's Health, 39(2), 381-388. https://doi.org/10.5534/wjmh.200152 
Hulsen, T., de Vlieg, J., \& Alkema, W. (2008). BioVenn - a web application for the comparison and visualization of biological lists using area-proportional Venn diagrams. BMC Genomics, 9(1), 488. https://doi.org/10.1186/1471-2164-9-488

Jänne, M., Deol, H. K., Power, S. G., Yee, S. P., \& Hammond, G. L. (1998). Human sex hormone-binding globulin gene expression in transgenic mice. Molecular Endocrinology (Baltimore, Md.), 12(1), 123-136. https://doi.org/10.1210/mend.12.1.0050

Keene, D. E., Suescun, M. O., Bostwick, M. G., Chandrashekar, V., Bartke, A., \& Kopchick, J. J. (2002). Puberty Is Delayed in Male Growth Hormone Receptor Gene-Disrupted Mice. Journal of Andrology, 23(5), 661-668. https://doi.org/10.1002/j.1939-4640.2002.tb02308.x

Kim, J., \& Coetzee, G. A. (2004). Prostate specific antigen gene regulation by androgen receptor. Journal of Cellular Biochemistry, 93(2), 233-241. https://doi.org/10.1002/jcb.20228

Korenbrot, C. C., Huhtaniemi, I. T., \& Weiner, R. I. (1977). Preputial Separation as an External Sign of Pubertal Development in the Male Rat1. Biology of Reproduction, 17(2), 298-303. https://doi.org/10.1095/biolreprod17.2.298

Lawrence, M. G., Lai, J., \& Clements, J. A. (2010). Kallikreins on Steroids: Structure, Function, and Hormonal Regulation of Prostate-Specific Antigen and the Extended Kallikrein Locus. Endocrine Reviews, 31(4), 407-446. https://doi.org/10.1210/er.2009-0034

Love, M. I., Huber, W., \& Anders, S. (2014). Moderated estimation of fold change and dispersion for RNA-seq data with DESeq2. Genome Biology, 15(12), 550. https://doi.org/10.1186/s13059-014-0550-8

Mani, S. K., Mermelstein, P. G., Tetel, M. J., \& Anesetti, G. (2012). Convergence of Multiple Mechanisms of Steroid Hormone Action. Hormone and Metabolic Research, 44(8), 569-576. https://doi.org/10.1055/s-0032-1306343

Meistrich, M. L., \& Hess, R. A. (2013). Assessment of Spermatogenesis Through Staging of Seminiferous Tubules. In D. T. Carrell \& K. I. Aston (Eds.), Spermatogenesis (Vol. 927, pp. 299-307). Humana Press. https://doi.org/10.1007/978-1-62703-038-0_27

Miyaso, H., Nagahori, K., Takano, K., Omotehara, T., Kawata, S., Li, Z.-L., Kuramasu, M., Wu, X., Ogawa, Y., \& Itoh, M. (2021). Neonatal maternal separation causes decreased numbers of sertoli cell, spermatogenic cells, and sperm in mice. Toxicology Mechanisms and Methods, 31(2), 116-125. https://doi.org/10.1080/15376516.2020.1841865

Pontén, F., Jirström, K., \& Uhlen, M. (2008). The Human Protein Atlas-A tool for pathology. The Journal of Pathology, 216(4), 387-393.

https://doi.org/10.1002/path.2440

Sahadevan, M., \& Kumar, P. G. (2021). Peptidyl Arginine Deiminase 2 (PADI2) is 
Expressed in Post-Meiotic Germ Cells in the Mouse Testis and is Localized Heavily on the Acrosomal Region of Spermatozoa. Journal of Endocrinology and Reproduction, 25(1), 37-49. https://doi.org/10.18311/jer/2021/27986

Singh, P., \& Schimenti, J. C. (2015). The genetics of human infertility by functional interrogation of SNPs in mice. Proceedings of the National Academy of Sciences, 112(33), 10431-10436. https://doi.org/10.1073/pnas.1506974112

Singh, P., Schimenti, J. C., \& Bolcun-Filas, E. (2015). A Mouse Geneticist's Practical Guide to CRISPR Applications. Genetics, 199(1), 1-15. https://doi.org/10.1534/genetics.114.169771

Takahara, H., Kusubata, M., Tsuchida, M., Kohsaka, T., Tagami, S., \& Sugawara, K. (1992). Expression of peptidylarginine deiminase in the uterine epithelial cells of mouse is dependent on estrogen. The Journal of Biological Chemistry, 267(1), 520-525.

Tsuji-Hosokawa, A., Kashimada, K., Kato, T., Ogawa, Y., Nomura, R., Takasawa, K., Lavery, R., Coschiera, A., Schlessinger, D., Harley, V. R., Takada, S., \& Morio, T. (2018). Peptidyl arginine deiminase 2 (Padi2) is expressed in Sertoli cells in a specific manner and regulated by SOX9 during testicular development. Scientific Reports, 8(1), 13263. https://doi.org/10.1038/s41598-018-31376-8

Valesini, G., Gerardi, M. C., lannuccelli, C., Pacucci, V. A., Pendolino, M., \& Shoenfeld, Y. (2015). Citrullination and autoimmunity. Autoimmunity Reviews, 14(6), 490497. https://doi.org/10.1016/j.autrev.2015.01.013

Varet, H., Brillet-Guéguen, L., Coppée, J.-Y., \& Dillies, M.-A. (2016). SARTools: A DESeq2- and EdgeR-Based R Pipeline for Comprehensive Differential Analysis of RNA-Seq Data. PLoS ONE, 11(6), e0157022. https://doi.org/10.1371/journal.pone.0157022

Vossenaar, E. R., Zendman, A. J. W., van Venrooij, W. J., \& Pruijn, G. J. M. (2003). $P A D$, a growing family of citrullinating enzymes: Genes, features and involvement in disease. BioEssays: News and Reviews in Molecular, Cellular and Developmental Biology, 25(11), 1106-1118. https://doi.org/10.1002/bies.10357

Wang, L., Song, G., Zhang, X., Feng, T., Pan, J., Chen, W., Yang, M., Bai, X., Pang, Y., Yu, J., Han, J., \& Han, B. (2017). PADI2-Mediated Citrullination Promotes Prostate Cancer Progression. Cancer Research, 77(21), 5755-5768. https://doi.org/10.1158/0008-5472.CAN-17-0150

Wang, S., \& Wang, Y. (2013). Peptidylarginine deiminases in citrullination, gene regulation, health and pathogenesis. Biochimica et Biophysica Acta, 1829(10), 1126-1135. https://doi.org/10.1016/j.bbagrm.2013.07.003

Wang, Y., Wysocka, J., Sayegh, J., Lee, Y.-H., Perlin, J. R., Leonelli, L., Sonbuchner, L. S., McDonald, C. H., Cook, R. G., Dou, Y., Roeder, R. G., Clarke, S., Stallcup, M. R., Allis, C. D., \& Coonrod, S. A. (2004). Human PAD4 Regulates Histone Arginine Methylation Levels via Demethylimination. Science, 306(5694), 279- 
283. https://doi.org/10.1126/science. 1101400

Witalison, E. E., Thompson, P. R., \& Hofseth, L. J. (2015). Protein Arginine Deiminases and Associated Citrullination: Physiological Functions and Diseases Associated with Dysregulation. Current Drug Targets, 16(7), 700-710.

Yeh, S., Tsai, M.-Y., Xu, Q., Mu, X.-M., Lardy, H., Huang, K.-E., Lin, H., Yeh, S.-D., Altuwaijri, S., Zhou, X., Xing, L., Boyce, B. F., Hung, M.-C., Zhang, S., Gan, L., Chang, C., \& Hung, M.-C. (2002). Generation and characterization of androgen receptor knockout (ARKO) mice: An in vivo model for the study of androgen functions in selective tissues. Proceedings of the National Academy of Sciences of the United States of America, 99(21), 13498-13503.

https://doi.org/10.1073/pnas.212474399

Yoshimura, S., Yamaguchi, H., Konno, K., Ohsawa, N., Noguchi, S., \& Chisaka, A. (2005). Observation of Preputial Separation is a Useful Tool for Evaluating Endocrine Active Chemicals. Journal of Toxicologic Pathology, 18(3), 141-157. https://doi.org/10.1293/tox.18.141

Zhang, X., Bolt, M., Guertin, M. J., Chen, W., Zhang, S., Cherrington, B. D., Slade, D. J., Dreyton, C. J., Subramanian, V., Bicker, K. L., Thompson, P. R., Mancini, M. A., Lis, J. T., \& Coonrod, S. A. (2012). Peptidylarginine deiminase 2-catalyzed histone $\mathrm{H} 3$ arginine 26 citrullination facilitates estrogen receptor a target gene activation. Proceedings of the National Academy of Sciences, 109(33), 1333113336. https://doi.org/10.1073/pnas.1203280109

Zhu, X., Xu, Y., Yu, S., Lu, L., Ding, M., Cheng, J., Song, G., Gao, X., Yao, L., Fan, D., Meng, S., Zhang, X., Hu, S., \& Tian, Y. (2014). An Efficient Genotyping Method for Genome-modified Animals and Human Cells Generated with CRISPR/Cas9 System. Scientific Reports, 4(1), 6420. https://doi.org/10.1038/srep06420

\section{FIGURE LEGENDS}

Figure 1. Design of Padi2/Padi4 double knockout mice. (a) Schematic of the CRISPR protocol using Padi2 KO zygotes. (b) Design and sequence of the guide RNA targeting Exon 1 of the Padi4 gene. (c) Sanger sequencing confirmed a four nucleotide deletion in the DKO Padi4 Exon 1, resulting in (d) a frame-shift mutation and premature stop codon in the predicted amino acid sequence.

Figure 2. Authentication of the Padi2/Padi4 double knockout. (a) RT-PCR of salivary gland tissue shows that the DKO mice lack Padi2 transcripts. (b) PCR analysis of genomic DNA (15\% polyacrylamide gel) confirms a 4bp deletion within the Padi4 gene of DKO mice. (c) Western blot analysis of spleen lysate confirms that DKO mice lack both PAD2 and PAD4 mature proteins. 
Figure 3. Breeding phenotype of Padi2/Padi4 double knockout mice. (a) In controlled breeding trials, DKO pairs appeared to take longer than WT to have their first litter and had fewer pup-producing pairs (data not significant). (b) Two days postpartum, DKO pups were significantly smaller than WT pups (one-tailed t-test, ${ }^{*} \mathrm{p}=0.004$ ). (c) Male DKO are significantly smaller than both SKO and WT mice at weaning (ANOVA, $p=0.02$, post-hoc one-tailed t-test with $W T,{ }^{* *} p=0.003$ ). (d) Male DKO weanlings are visibly smaller and less developed than WT and SKO weanlings.

Figure 4. Pubertal onset is delayed in Padi2/Padi4 DKO male mice. DKO males took an average of 49 days to undergo preputial separation (PS) compared to 46 days for WT males ( $n=46$, two-tailed t-test, $\left.{ }^{*} p<0.00001\right)$.

Figure 5. Padi2/Padi4 DKO male mice show altered serum hormone levels. (a) Serum Testosterone levels were lower in DKO males when compared to WT males at all timepoints, reaching significance at day 48 . (b,c) LH and FSH levels were significantly higher in DKO on day 50 , then dropped to values that were significantly lower than WT by day $90\left(n=5\right.$, one-tailed t-test, $\left.{ }^{*} \mathrm{p}=0.05,{ }^{* *} \mathrm{p}=0.02\right)$.

Figure 6. Padi2/Padi4 DKO male mice have disrupted testicular development. (a) Total testes weight was significantly lower in 90-day old DKO mice compared to WT $\left(n=5\right.$, one-tailed t-test, $\left.{ }^{*} p=0.0005\right)$. (b) Hematoxylin and Eosin stained sections of WT testis showing normal spermatogenic cell structures. (c-e) Hematoxylin and Eosin stained sections of DKO testis. Black arrows indicate: (c) apoptotic spermatocytes, (d) apoptotic spermatogonia and (e) atypical residual bodies. (scale bars $=50 \mu \mathrm{m}$ ).

Figure 7. RNA-seq analysis of gene expression in WT and DKO testes. (a) Venn diagram of differential gene expression shows that 263 genes are significantly upregulated in DKO in comparison to WT, with only 17 genes overlapping the P2KO profile. Gene lists are provided in Supplemental 3 and 4 . (b-c) Gene ontology analysis with DAVID 6.8 divides these upregulated genes into (b) Biological Processes with reproductive pathways heavily represented and (c) Molecular Functions almost entirely represented by genes involved in nucleic acid regulation.

Figure 8. A heatmap and hierarchical dendrogram illustrates the top 100 differentially expressed genes between WT, P2KO and DKO. Red=upregulated, Blue=downregulated. The list of DEGs used to create the heatmap is provided in Supplemental 6 . 


\section{Figure 1}

(a) P2KO

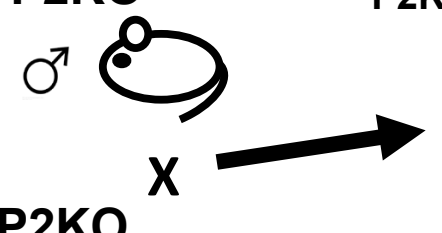

P2KO zygotes

Fo

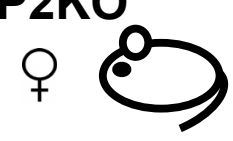

CRISPR injection (Cas9 and Padi4 sgRNA)

(Padi4 het)

(b)

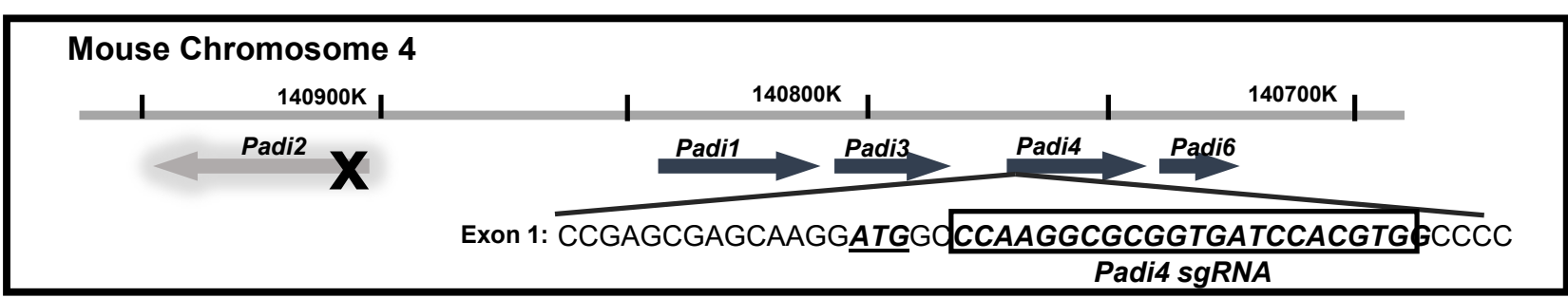

(c) WT GCGAGCAAGg ATG GCCCAAGGCGCGgTGATCCACGTGGCCCCCGAGCAGCCCACTCAC DKO GCGAGCAAGG

(d) WT Met AQGAVIHVAPEQPTHAVCVVGTATPLDVRGSAPKGYTTFGITASPGVIVDVIHGPPVK... DKO Met AQGAVIAPPPSSPLTPCVWWAPRPRWMSAVLLLRATQPSASQPLQESS Stop 
Figure 2

(a)

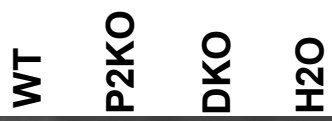

mPadi2

GAPDH

(b)

200 bp

150 bp

(c)
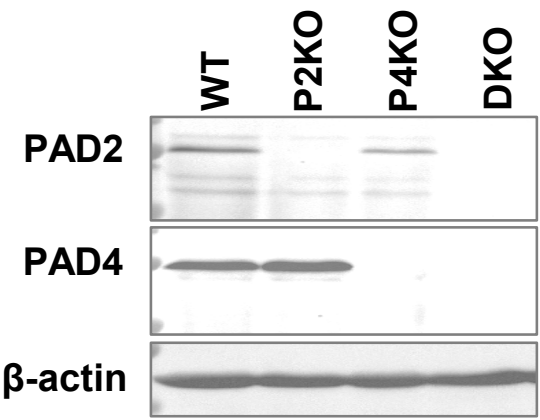

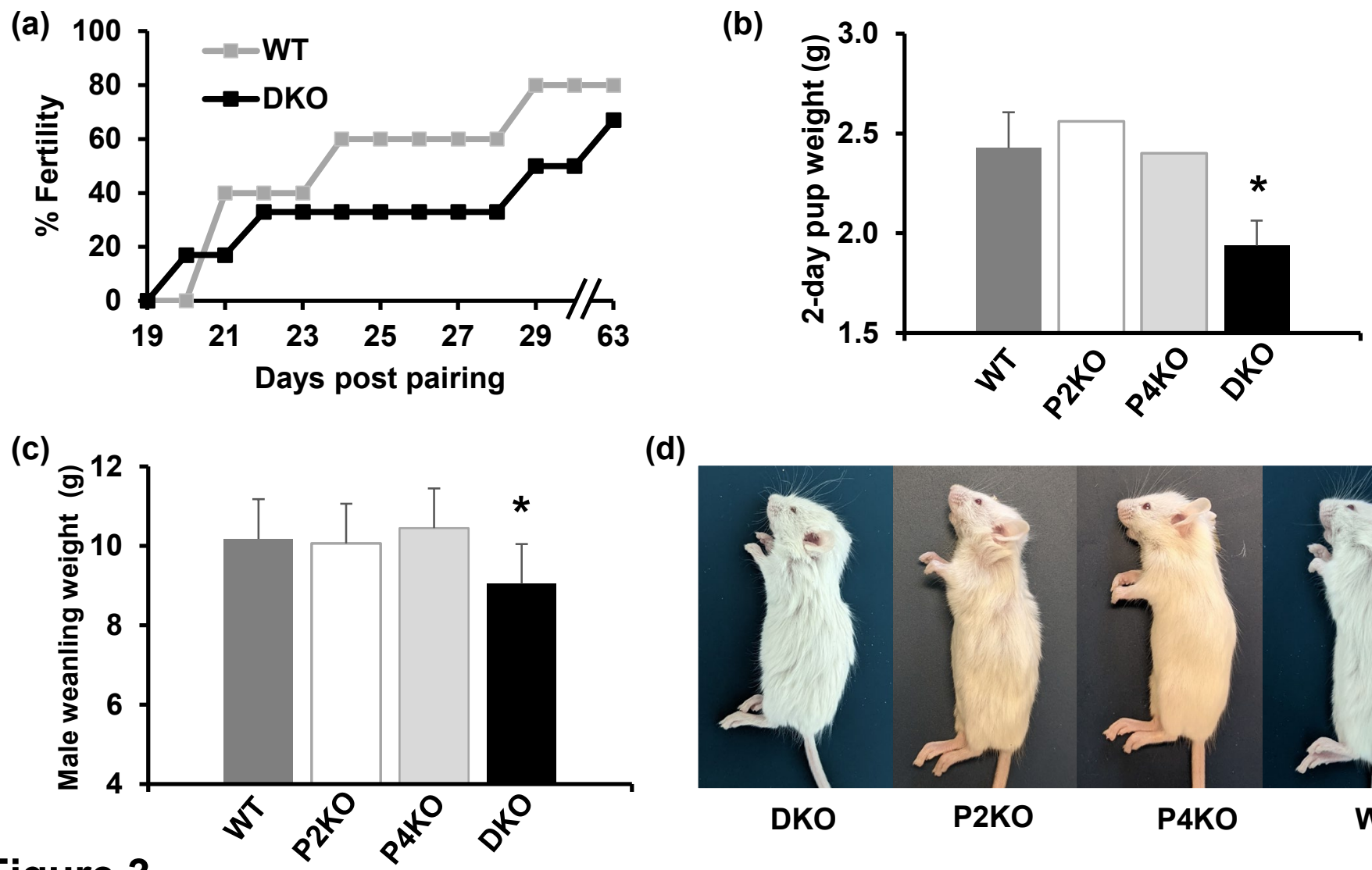

(d)

Figure 3

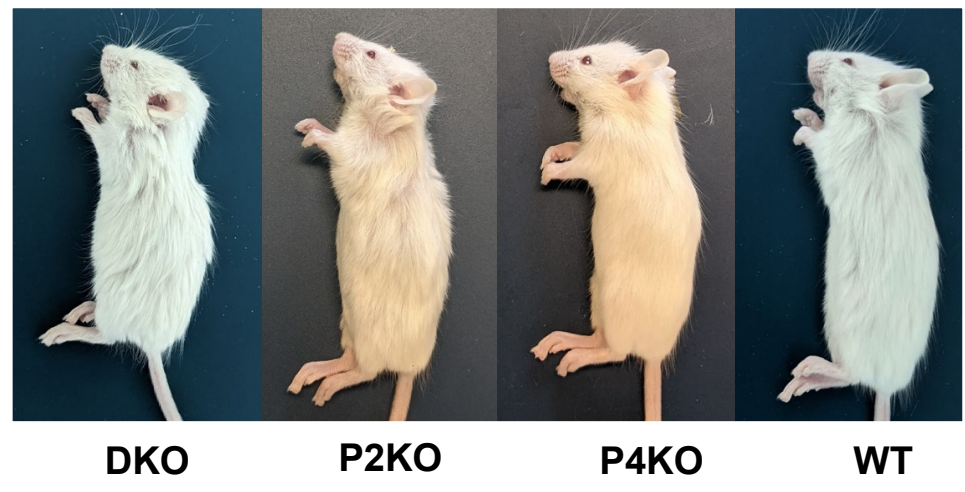




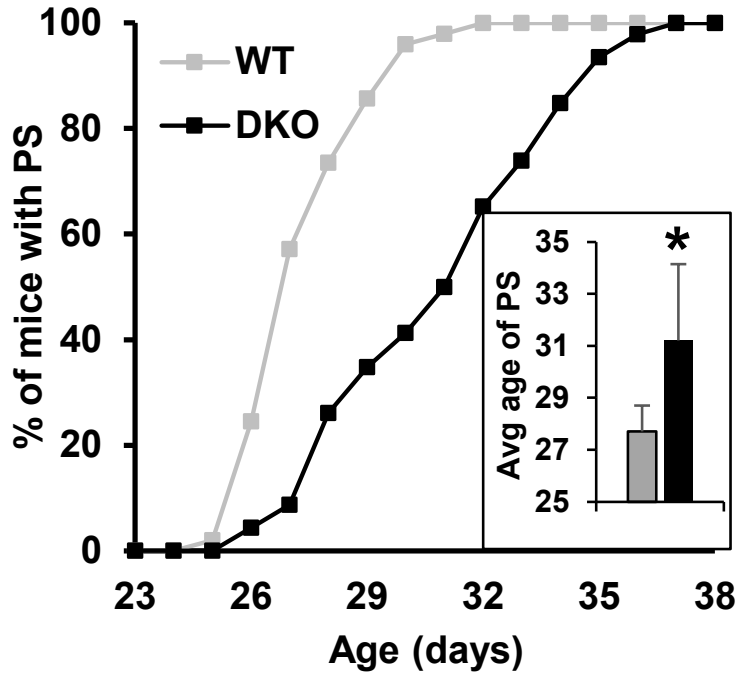

Figure 4 
(a) Figure 5

Testosterone

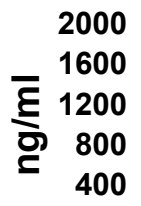

(b) 25

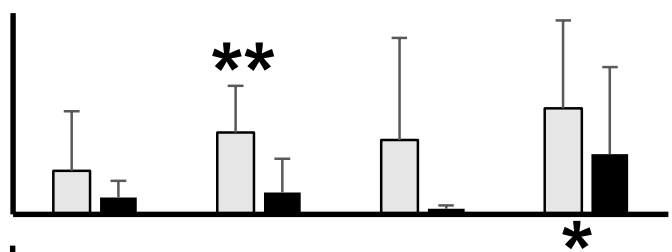

$\begin{array}{ll}\bar{E} & 20 \\ \text { 马े } & 15 \\ \text { द } & 10\end{array}$

5

(c)

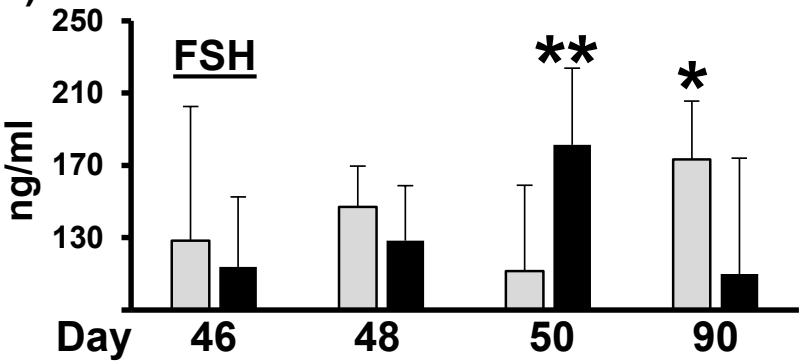


(a)

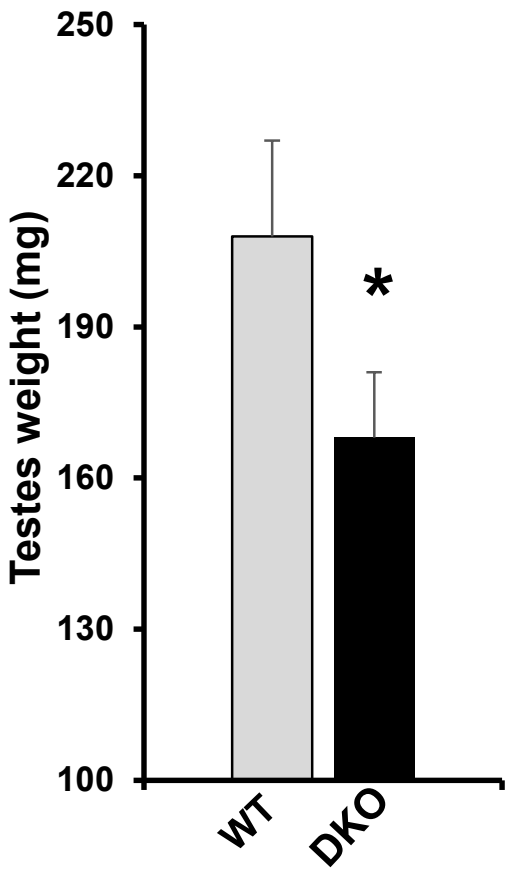

(b)

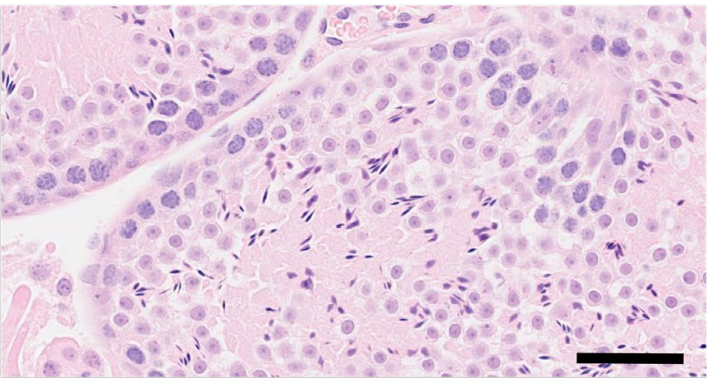

(d)

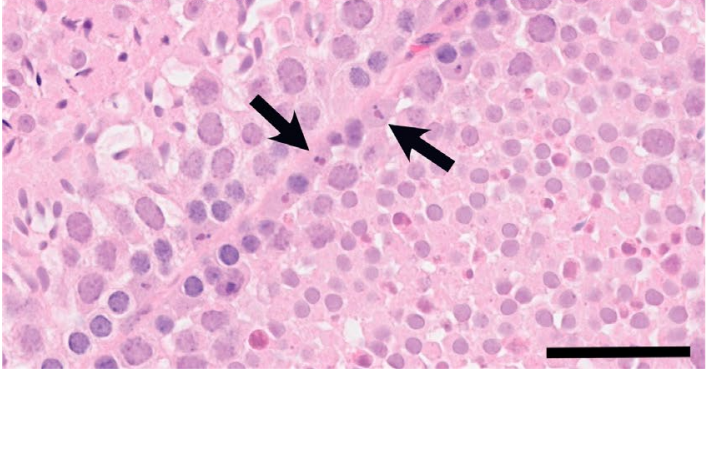

(c)

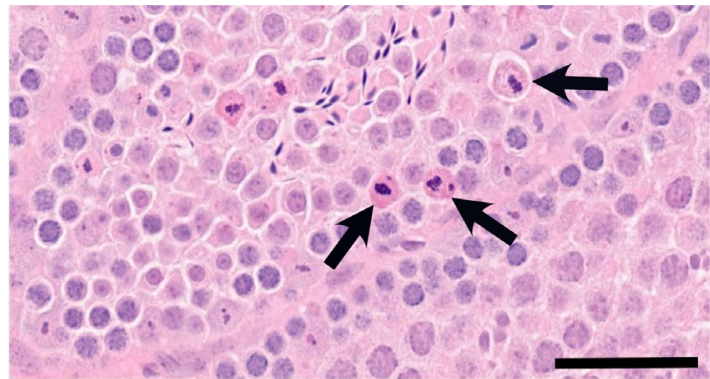

(e)

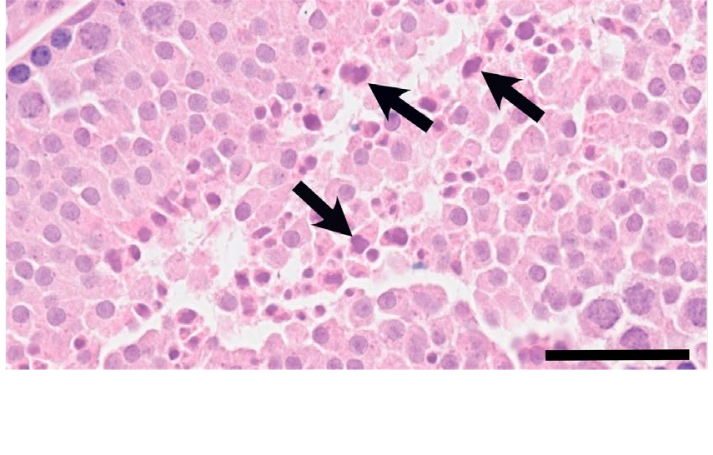

Figure 6 


\section{(a) Genes upregulated compared to WT}

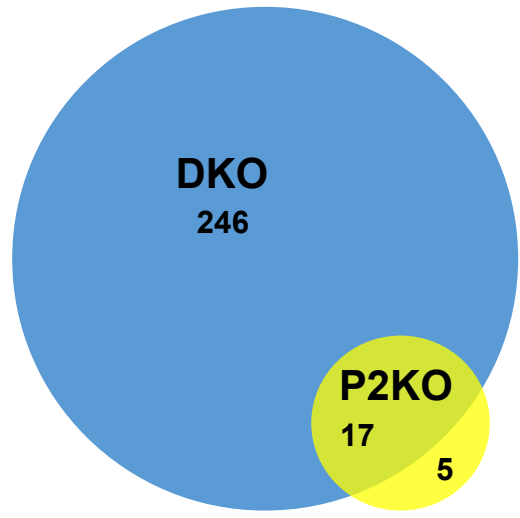

(b) Gene Ontology - Biological Processes

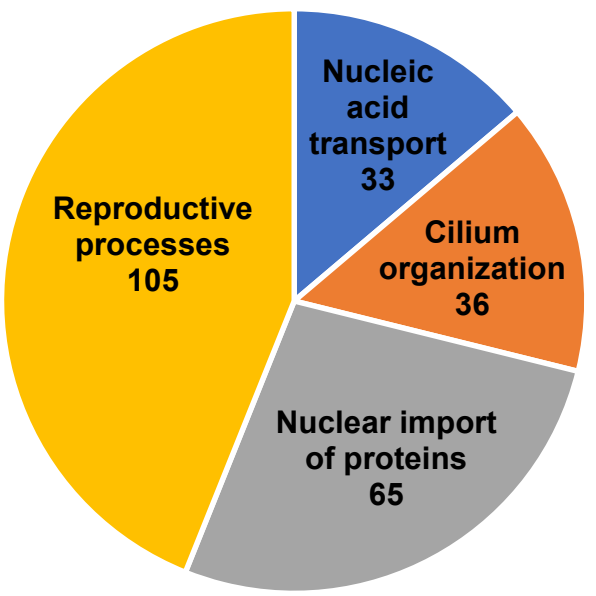

(c) Gene Ontology - Molecular Function

\section{Figure 7}

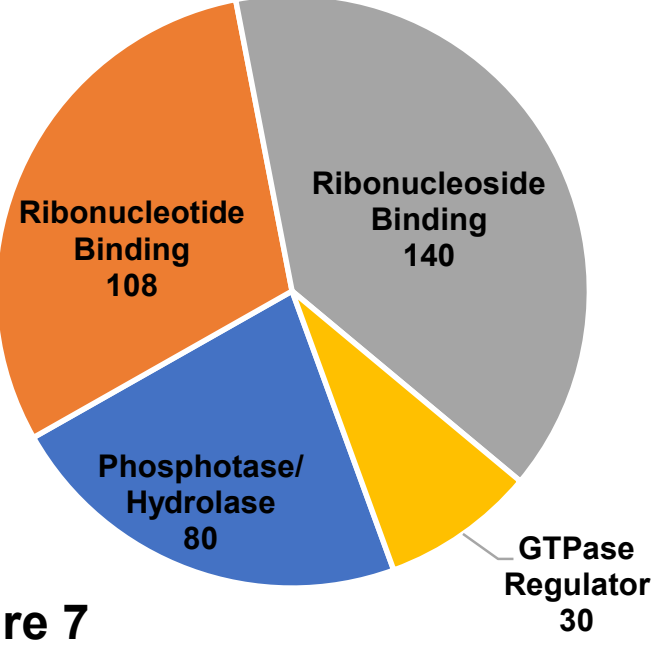




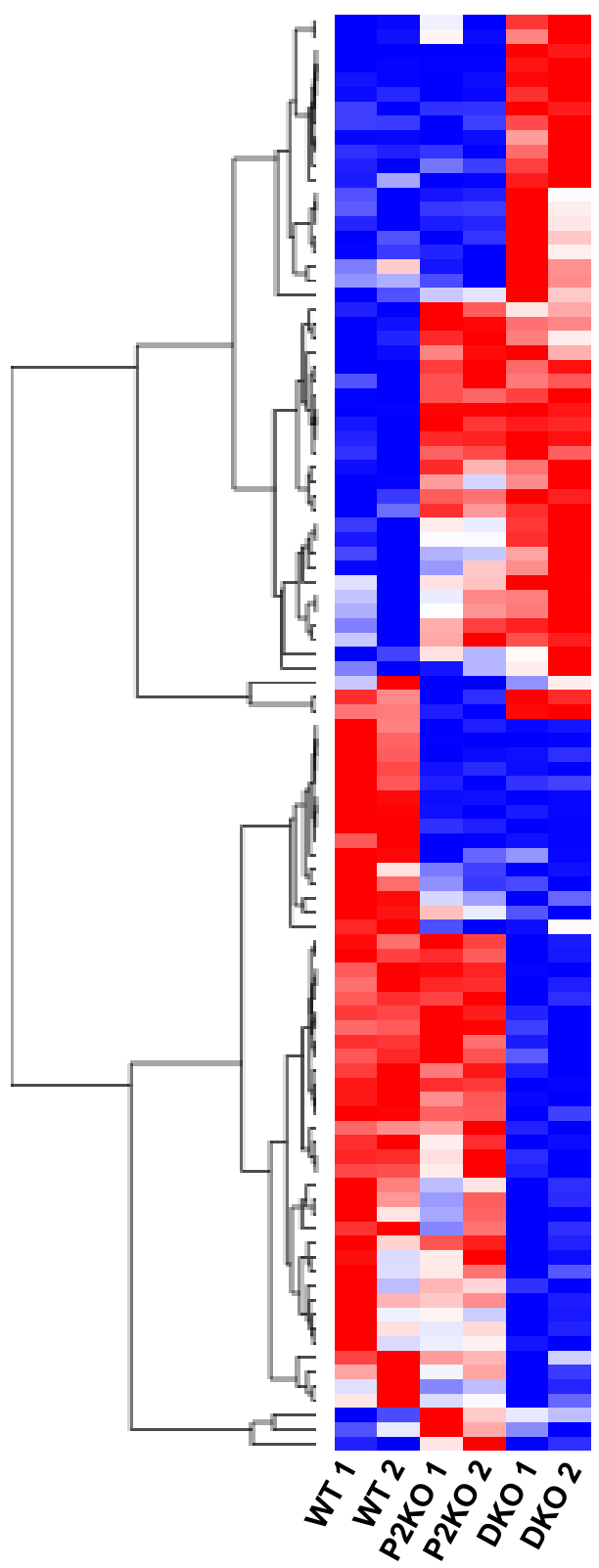

Figure 8 